\title{
DESIGN AND FABRICATION OF COMPACT AND High PERFormanCE C BAND OMT
}

\author{
Rahul G \\ Antenna Products \& Satcom Division, Electronics Corporation of India Limited, India
}

\begin{abstract}
This paper presents the design, simulation and fabrication of simple $C$ band orthomode transducer (OMT) machined in two identical parts for manufacturing easiness. This device offers compact size and high electrical performance. The device consists of a common circular input port and rectangular ports for horizontal and vertical polarization separation. The common port has a direct transition from circular to rectangular waveguide for the through port coupling. The side port coupling is done via coupling slot and waveguide transition. The design of the device utilises the concept of class I asymmetric based OMT and intermediate truncated circular waveguide. The device is simulated and optimised in CST Microwave Studio and the simulated results of the device are compared with the measured results. The measured return losses of the both rectangular ports are better than $20 \mathrm{~dB}$, port to port isolation greater than $50 \mathrm{~dB}$ and cross-polarization discrimination better than $35 \mathrm{~dB}$.The measured results were in good agreement with the simulated results.
\end{abstract}

\section{KEYWORDS}

Orthomode Transducer, Compact, C Band, Isolation, Polarisation, Boifot, classI

\section{INTRODUCTION}

An Orthomode Transducer (OMT) is a basic component of feed system which provides the frequency reuse in earth station antennas. The OMT separates the orthogonal polarization within the same frequency band which enhances the channel capacity. There is an increase in demand for antennas with wide $\mathrm{C}$ band reception i.e. from 3.4-4.2 $\mathrm{GHz}$ by upgrading the existing antennas of frequency range 3.625-4.2 GHz. This requires the design change for enhancing the fractional bandwidth of OMT to $21 \%$. An asymmetric OMT is sufficient to cover less than $30 \%$ bandwidth to dictate the requisite port to port isolation and input matching capability without the introduction of higher order modes. In this paper the OMT is manufactured in two symmetrical pieces. A compact transition is employed from circular common port to rectangular through port through intermediate truncated circular waveguide [1]. Rectangular coupling slot with rectangular waveguide transition and iris is employed for the side port. The circular waveguide contain dual polarised signals of TE11 and TE*11 modes and the rectangular ports contain TE10 mode. The dimension of the circular waveguide is selected to allow the propagation of TE11 mode for the lowest frequency of operation. The two rectangular ports are having standard dimensions of WR229 to allow the fitment of low noise amplifier directly to the OMT. This paper covers the recent developments, description, design, simulation and fabrication of OMT. The entire simulation was performed and optimised in CST Microwave Studio. The OMT manufactured is then measured for RF performance. 


\section{REVIEW OF LITERATURE AND RELATED WORKS}

For a waveguide device broad band characteristics is achieved by symmetry. But the fact is that both symmetric and asymmetric devices generate higher order modes. Normally higher order modes are evanescent. But the higher order mode generated stores energy which degrades broad band characteristics. The even order higher order mode generated are easy to compensate compared to the odd order modes. Boifot basically classified waveguide OMTs as class I, II, and III [2],[3] based on their symmetry.

In class I implementation [4]-[7] the through port is symmetric which is used for one mode and a non symmetric side arm which is used for other mode. This is depicted as shown in Figure 1. Because of the non symmetry the percentage bandwidth support is nearly $20 \%$, due to the excitation of higher order modes. This OMT offers the best candidate for narrow bandwidth operation and simple fabrication.
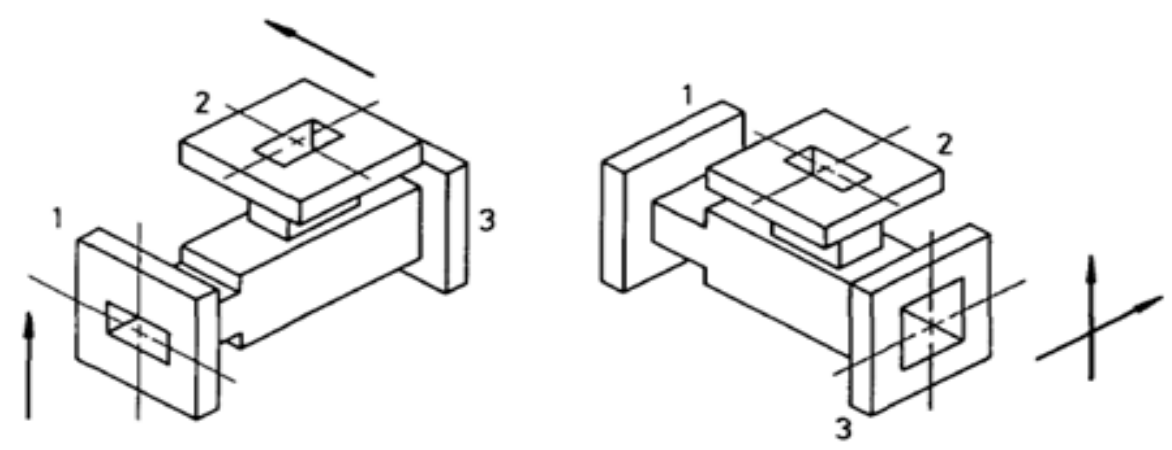

Figure 1: Basic outline of Class I OMT (from Schlegel and Fowler [8])

In class II implementation the side port is symmetrical by splitting two symmetrical arms from the common port and the through port is direct transition from the common port. This is depicted as shown in Figure 2. This OMT offers good broad band match performance even for side port.

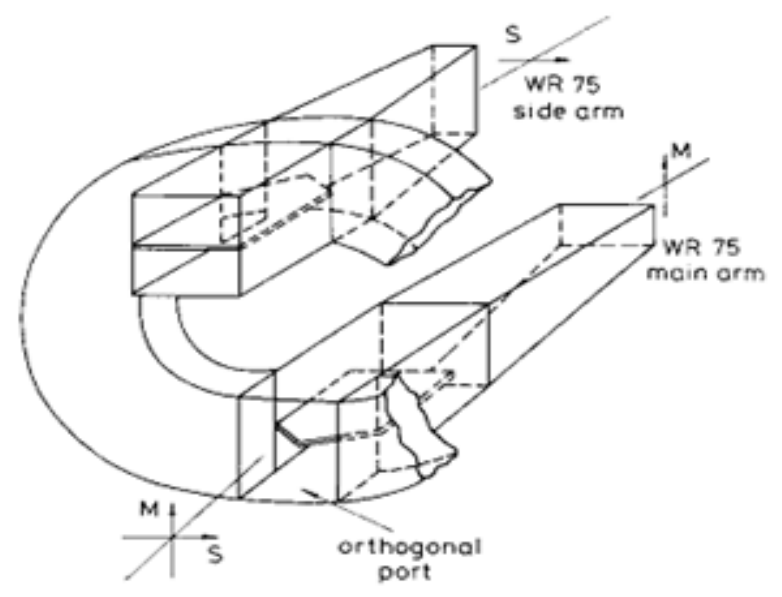

Figure 2: Basic outline of Class II OMT (from Brain [9]) 
In class III implementation both the arms are symmetrical [10]-[14] as shown in the Figure 3 below. It offers the natural higher order mode cancellations in both the ports. This cancellation is due to the combining of two ports via a power combiner. Thus class III offers the best broad band and isolation performance.

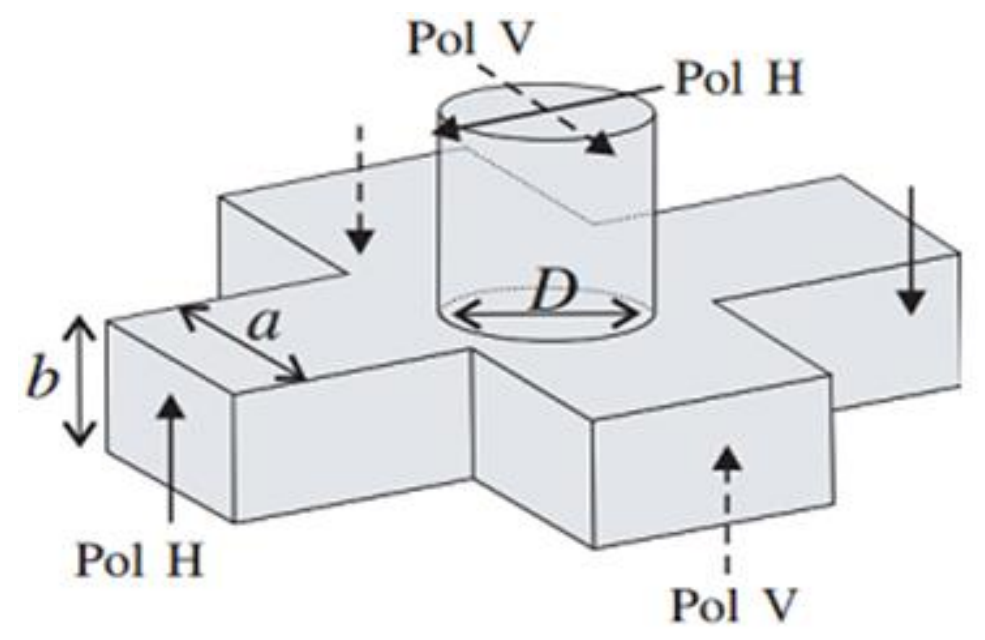

Figure 3: Basic outline of Class III OMT (Turnstile junction)

From the available literature since our required percentage bandwidth is only $21 \%$, a simple Class I type OMT is sufficient. From the previous works available [15],[16] the single piece fabrication of OMT having taper transition or with quarter wave transformers with reduced height rectangular waveguides is little complex and the overall length is also more. The most recent work [17] of a split type OMT with a common square port has the limitations of return loss and followed a gradual tapering from square common port to rectangular through port which makes the structure lengthier. So a combination of split type OMT with truncated circular waveguide and circular common port is being analysed in this paper.

\section{DESCRIPTION OF THE OMT}

The OMT consists of a common circular port of diameter $58.2 \mathrm{~mm}$ which can be directly attached to a $\mathrm{C}$ band feed horn. The two rectangular ports are of WR 229 dimensions to which $\mathrm{C}$ band LNA can be direct mounted. The OMT fabrication is done with two identical split pieces and is screwed at the centre portion to provide perfect butting for avoiding any gaps. The through port is cut from the center of broad wall of waveguide and side port cut from the middle of narrow wall of waveguide. At the middle of broad wall of waveguide, voltage is maximum, and current is minimum, so splitting from middle of broad wall does not affect the performance. On the other hand, at the middle of narrow wall of waveguide, voltage is minimum, and current is maximum, so splitting from middle of narrow wall disturbs the current distribution, hence it degrades the performance at side port. Thus performance of side port depends on the surface smoothness of the symmetrical halves. Deviations in the measured results of OMT from the simulated values may be attributed to fabrication and integration tolerance. The main specifications of the OMT are as shown in below Table 1. 
Table 1: OMT Specifications

\begin{tabular}{|l|l|}
\hline Parameters & Specification \\
\hline Frequency & $3.4-4.2 \mathrm{GHz}$ \\
\hline Number of Receive Port & 2 No's with WR229 interface \\
\hline VSWR & $1.3: 1$ max (Return loss better than $17.7 \mathrm{~dB})$ \\
\hline Polarization & Linear- H \&V \\
\hline Cross Polarisation Discrimination & Better than $35 \mathrm{~dB}$ \\
\hline Port to Port Isolation & Better than $50 \mathrm{~dB}$ \\
\hline
\end{tabular}

\section{DESIGN AND ANALYSIS OF OMT}

The design of the common port circular waveguide diameter is arrived at the TE11 propagation mode at the Common Port of OMT at the lowest frequency of operation ie, 3.4 GHz. The cut-off diameter of $58.2 \mathrm{~mm}$ corresponds to $\mathrm{f}=3.02 \mathrm{GHz}$ in TE11 mode.

The circular diameter has been matched to the broad wall dimension of the rectangular through port to simplify the manufacturing. The common port is followed by transition from circular to rectangular with waveguide mode converter/ waveguide transition which convert one of the dominant TE11 mode to TE10 mode of the through port i.e.,P1 as shown in Figure 2 below.

The waveguide transformers are basically $\lambda / 4$ transitions which are in the form of truncated circular waveguide. The usage of this transition makes the overall length of the OMT very compact and offers good electrical performance in terms of matching and insertion loss. The side port i.e., $\mathrm{P} 2$ couples the TE*11 mode and convert to TE*10 horizontally polarized waves through the rectangular coupling slot. The transformers in the direct path of the OMT offers virtual short for the TE*11 modes, hence allowing maximum coupling of power from this mode to the side port is achieved. The Side port coupling slot dimensions such as slot length and slot width are approximately $\lambda / 2$ and $\lambda / 10$ respectively. The matching sections consisting of quarter wave transformers from the coupling slots to WR229 dimensions. The inductive irises are introduced in the side port for providing matching impedance compensation to the capacitive effect of truncated circular section, hence improving the overall return loss. The placement of coupling slot is optimized in CST microwave studio for providing the maximum power coupling.

The entire structure is optimized in CST microwave studio to achieve the required specifications. The 3D model of the simulated OMT is shown in Fig 2 below.

The overall length of this compact OMT is $165 \mathrm{~mm}$. For the simulation set up three ports are defined with number of modes in the 


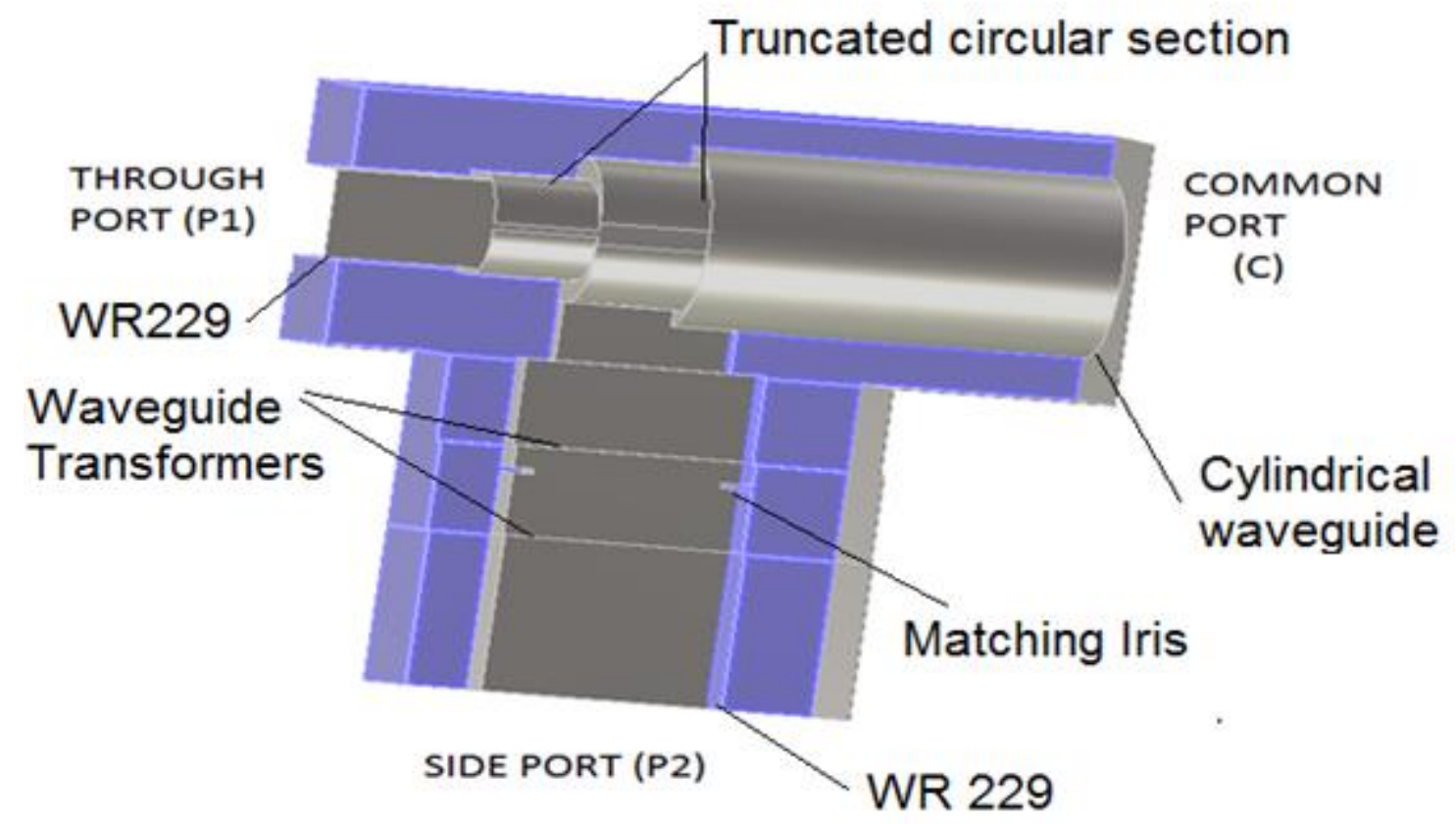

Figure 2: Detailed split section model of the proposed C band OMT

The optimized simulation result for the return loss for the side port is as shown in the Figure 3. It is observed that return losses are better than $22 \mathrm{~dB}$.

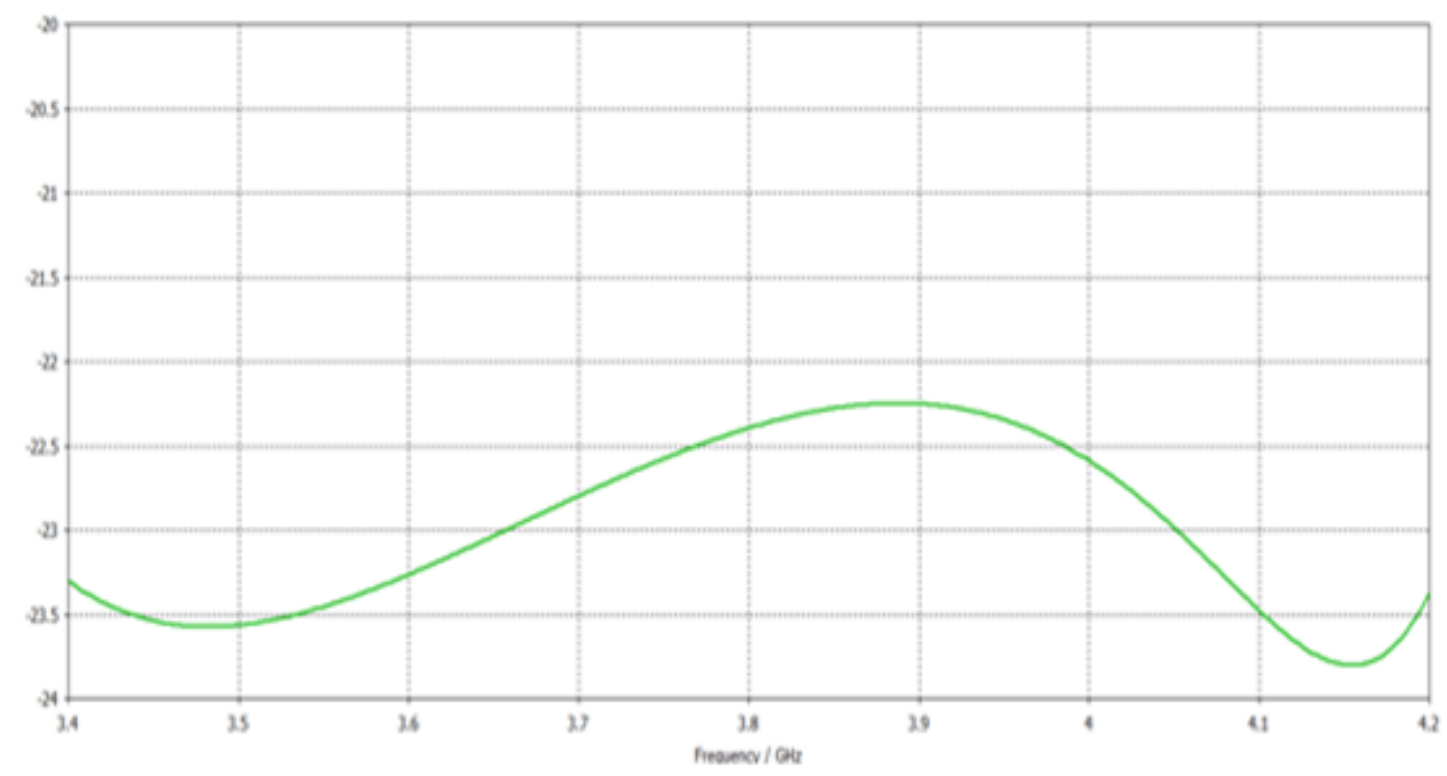

Figure 3: Simulated return loss port of side port (P2) plot

The optimized simulation result for the return loss for the through port is as shown in the Figure 4. It is observed that return losses are better than $25 \mathrm{~dB}$. 
International Journal Of Microwave Engineering (JMICRO) Vol.4, No.3/4, October 2019

SIMULATED RETURN LOSS OF P1

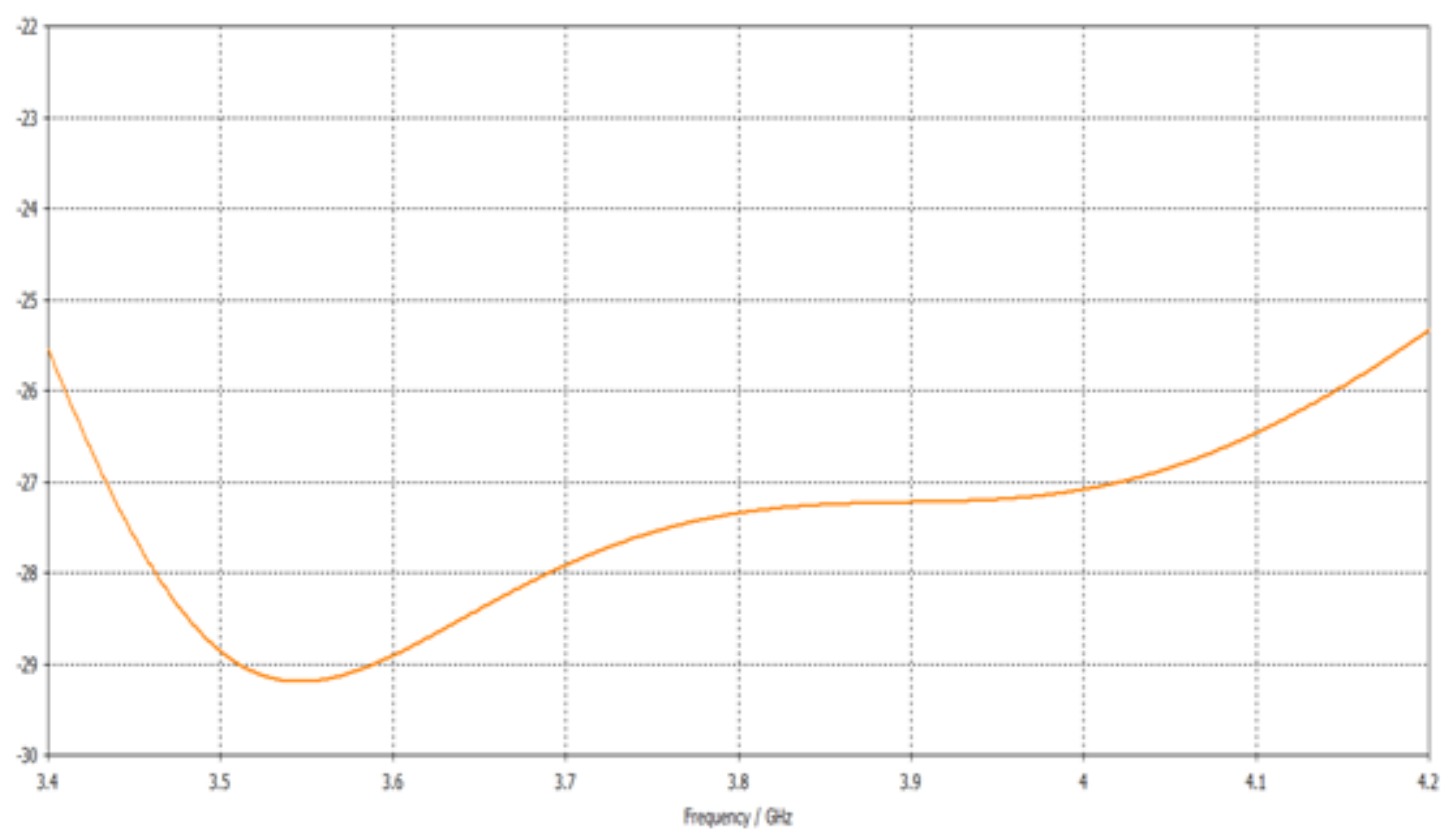

Figure 4: Simulated return loss port of through port (P1) plot

The optimized simulation result for the port to port isolation (between the two rectangular ports) is as shown in the Figure 5. It is noted that isolation value is better than $89 \mathrm{~dB}$.

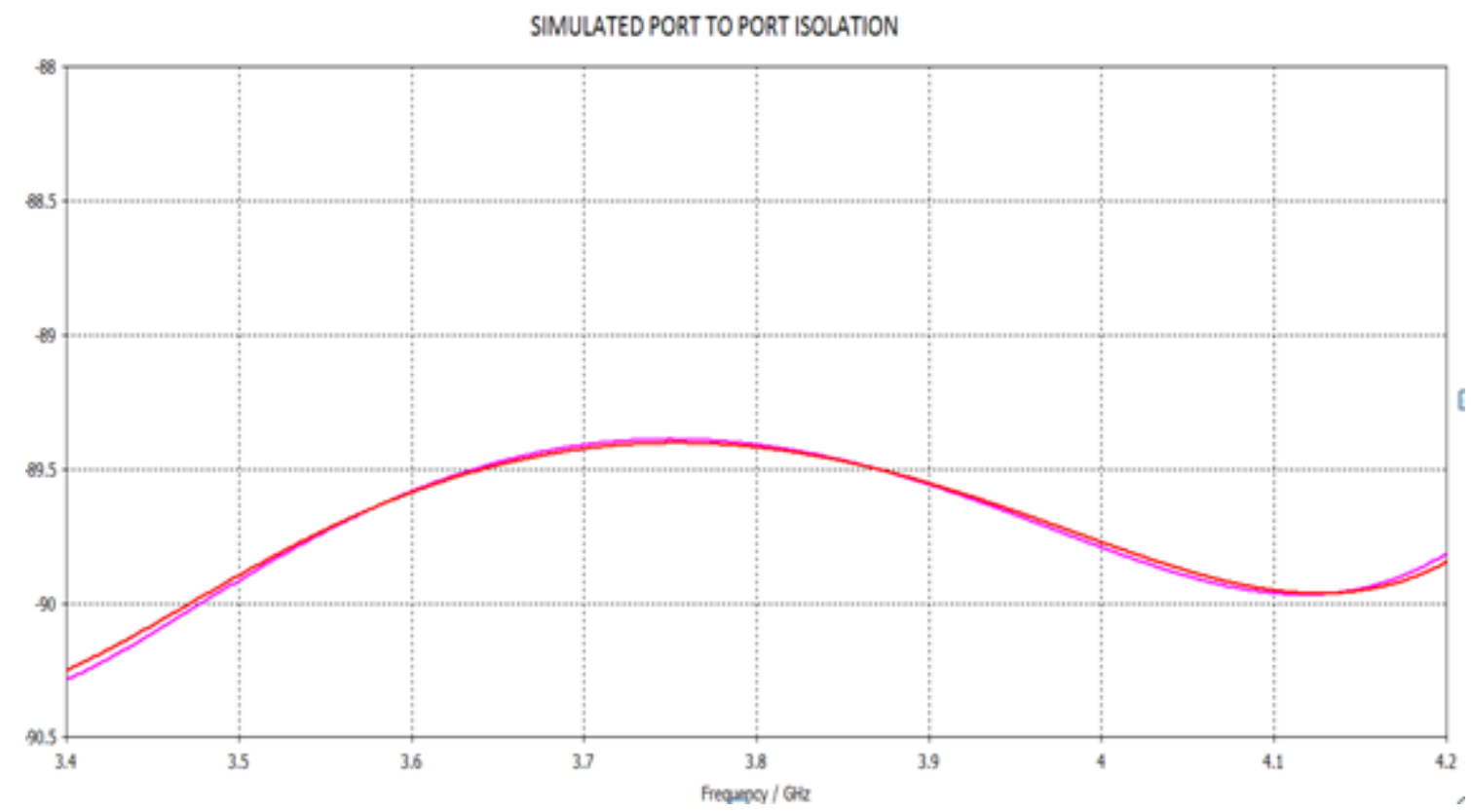

Figure 5: Simulated port to port isolation plot

The optimized simulation result for the cross polarization discrimination is as shown in the Figure 6. It is observed that the CPD values are better than $47 \mathrm{~dB}$. 


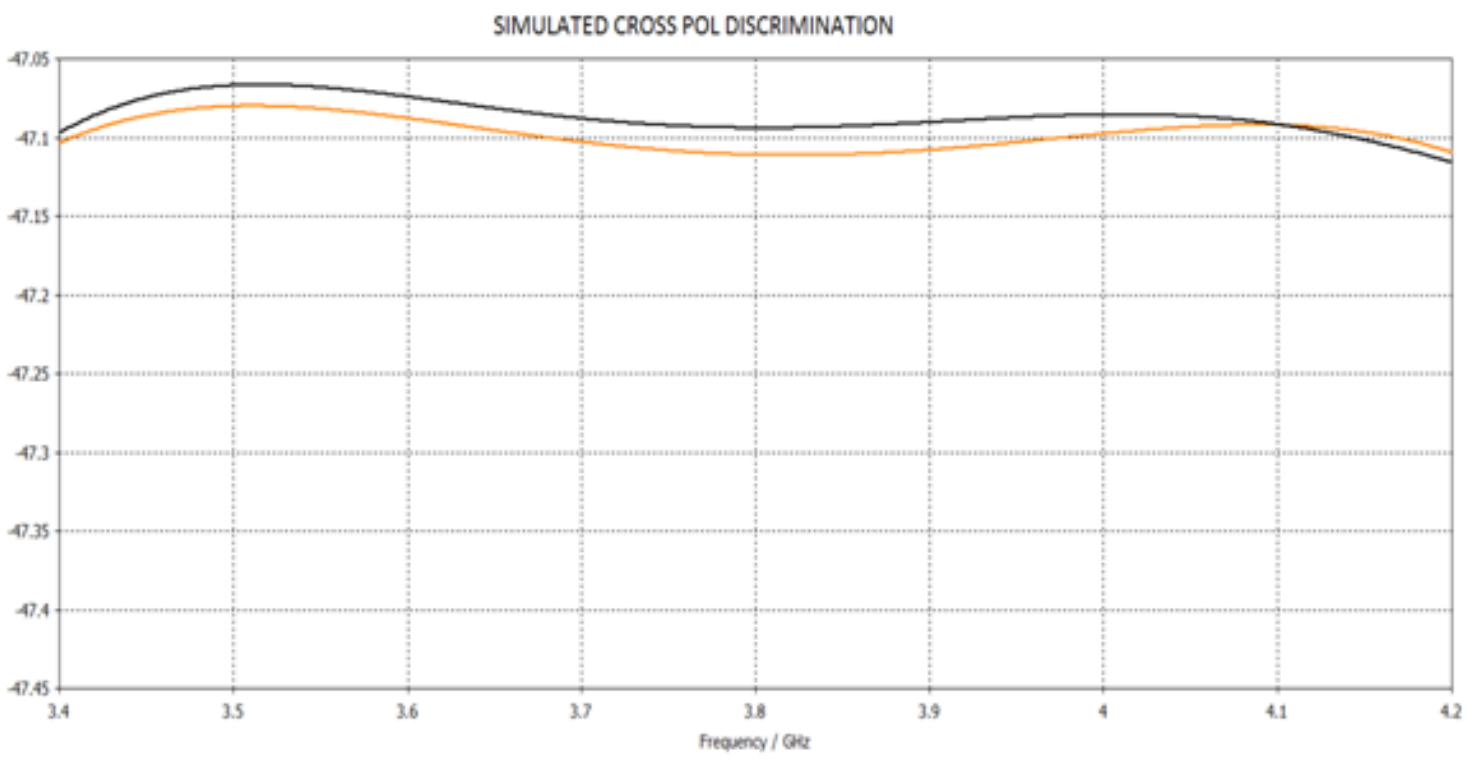

Figure 6: Simulated cross polarisation discrimination plot

The optimized simulation result for the insertion loss from the common port to the two orthogonal ports is as shown in the Figure 6. It is observed that the insertion loss values are less than $0.05 \mathrm{~dB}$.

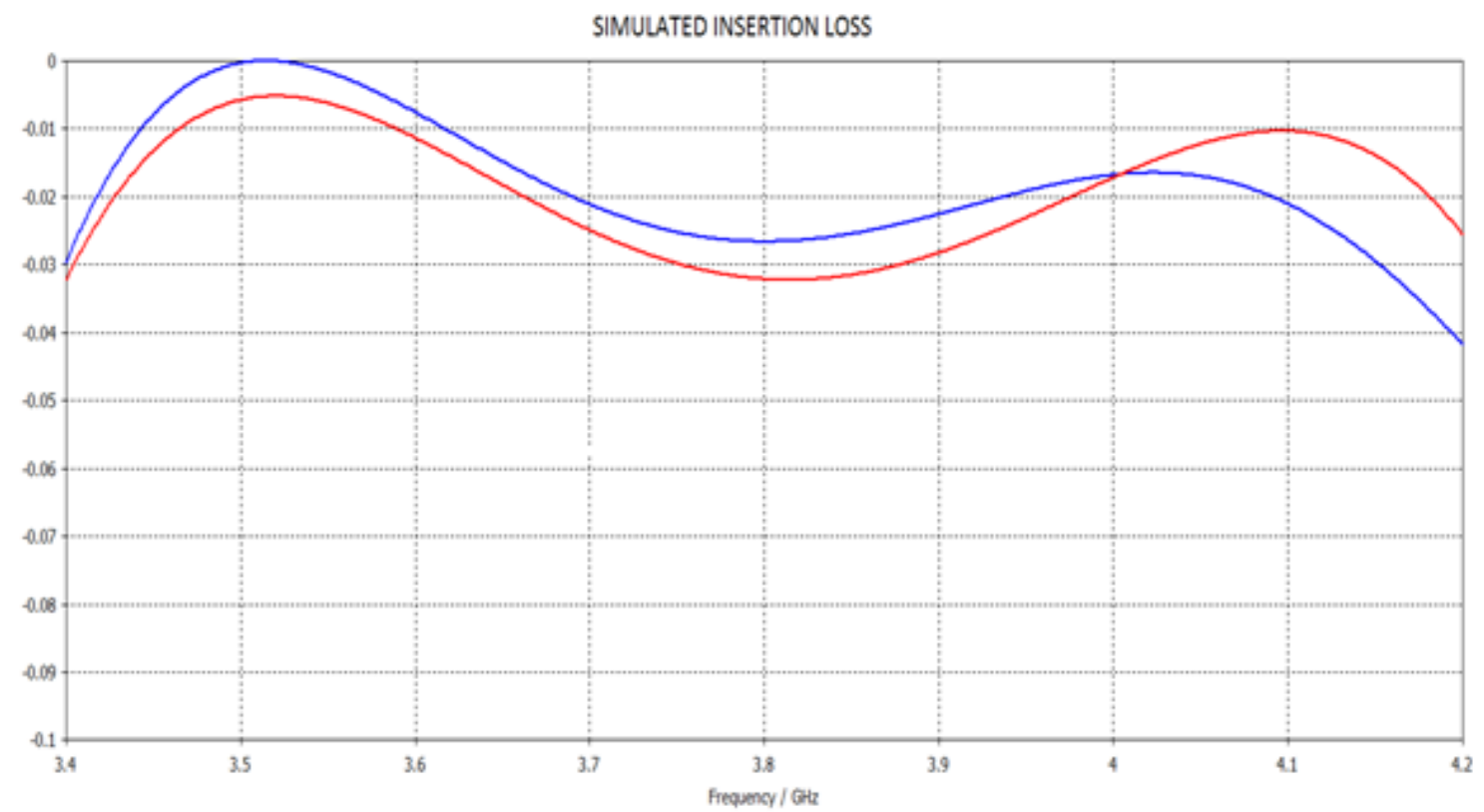

Figure 6: Simulated insertion loss plot

\section{MEASURED RESULTS OF THE OMT}

The OMT made out of aluminium is machined using CNC milling machine and wire cut. After manufacturing the OMT chromation is done for providing corrosion resistance. The two symmetrical pieces are screwed together and ensured that the surface smoothness is good enough to allow perfect butting. The ready to test OMT is as shown below in Figure 7. 


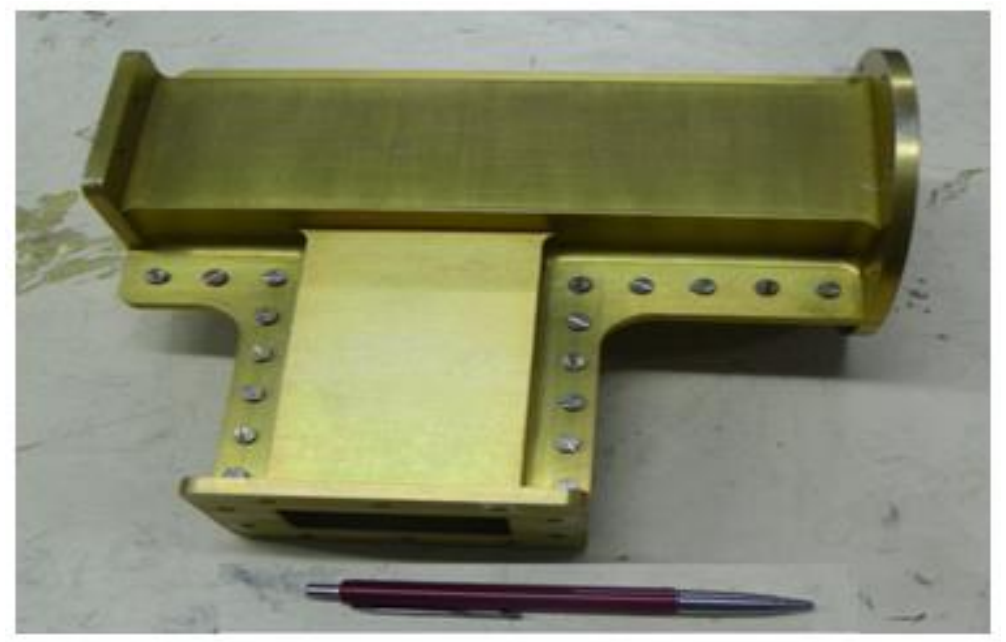

Figure 7: Fabricated OMT

The manufactured OMT is tested for the return loss, isolation, cross polarisation discrimination. The insertion loss of OMT cannot be measured to the non availability of one more OMT (back to back testing). The anechoic chamber within the lab is used for measurement of cross polarisation discrimination by having a rotatable probe.

The return loss of the OMT is measured with a scalar network analyser with unused port terminated. The Figure 8 below shows the measured return loss at the side port of the OMT with the other two ports terminated.

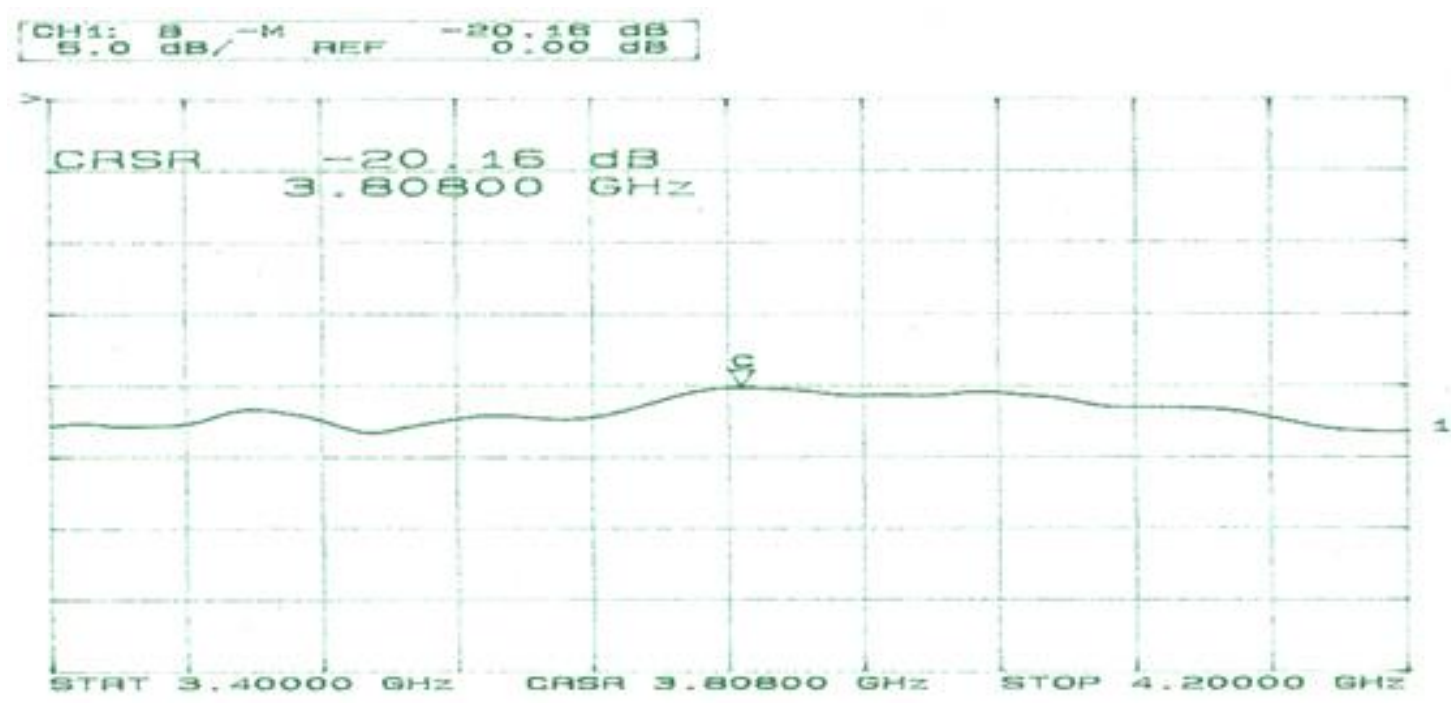

Figure 8: Measured return loss of side port (P2)

Similarly the return loss of through port is measured and recorded as shown in Figure 9 below. 
International Journal Of Microwave Engineering (JMICRO) Vol.4, No.3/4, October 2019

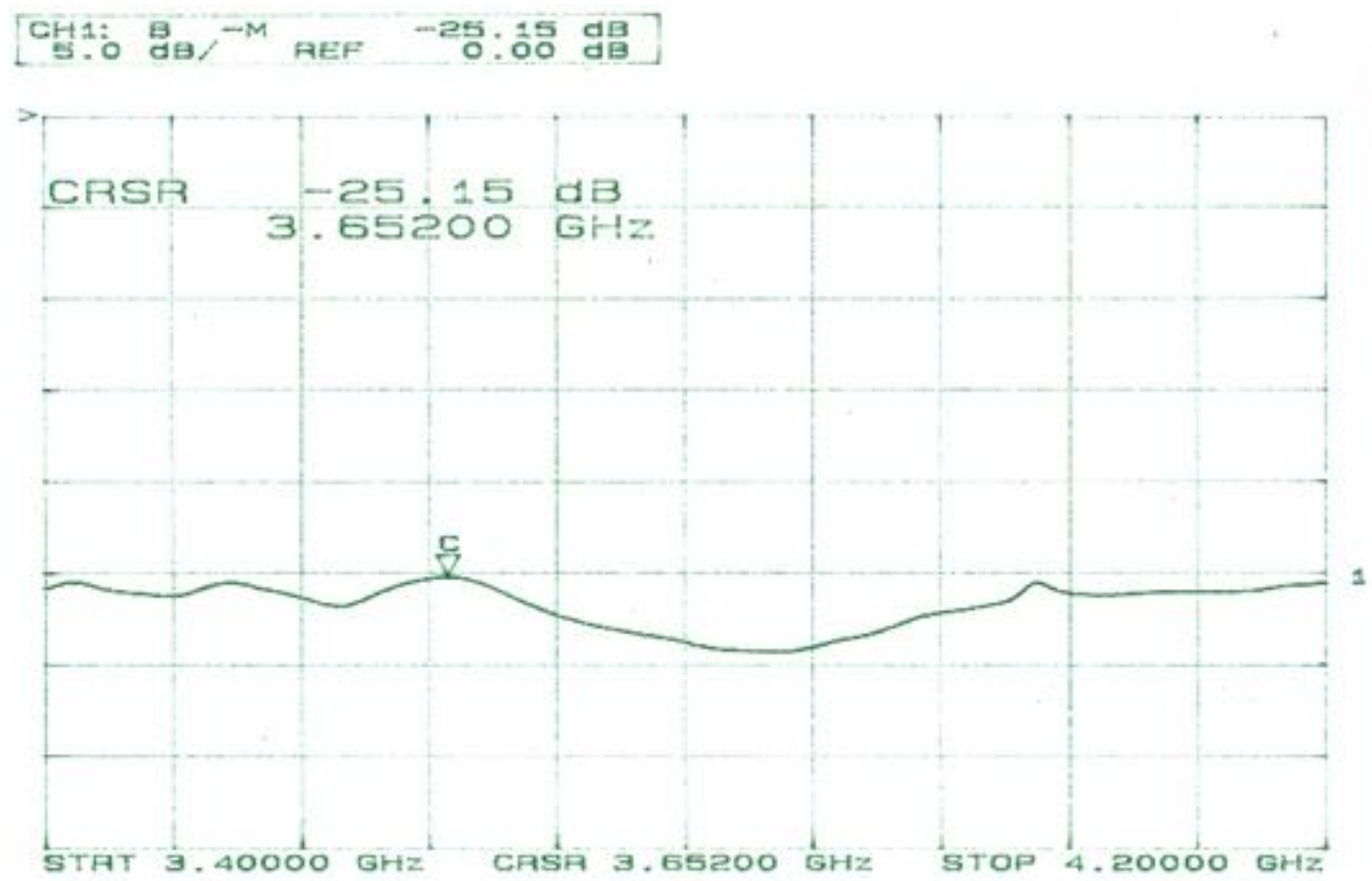

Figure 9: Measured return loss of through port (P1)

The Port to port isolation between the rectangular ports are measured by source at any one rectangular port and then receive at other rectangular port with the common port terminated with a circular load. Similarly the return loss of through port is measured and recorded as shown in Figure 9 below.

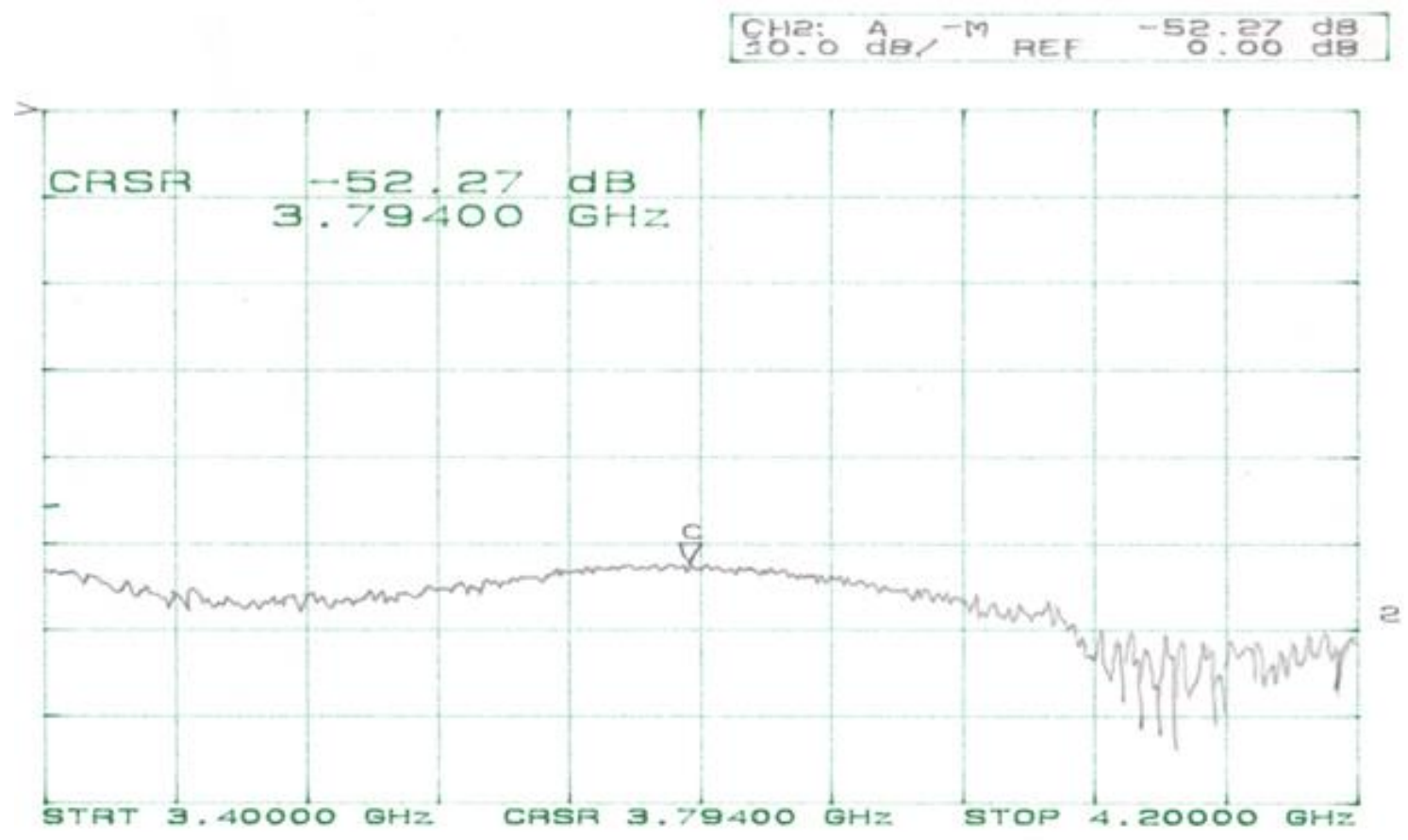

Figure 10: Measured port to port isolation 
International Journal Of Microwave Engineering (JMICRO) Vol.4, No.3/4, October 2019

Cross polarization for a given port is the amount of signal present from the orthogonal polarization. The measurement is done in a anechoic chamber with open ended waveguide as the source and the OMT ports as the receiver. The Figure 10 and 11 below shows the measured values at ports when excited with a source of orthogonal polarization.

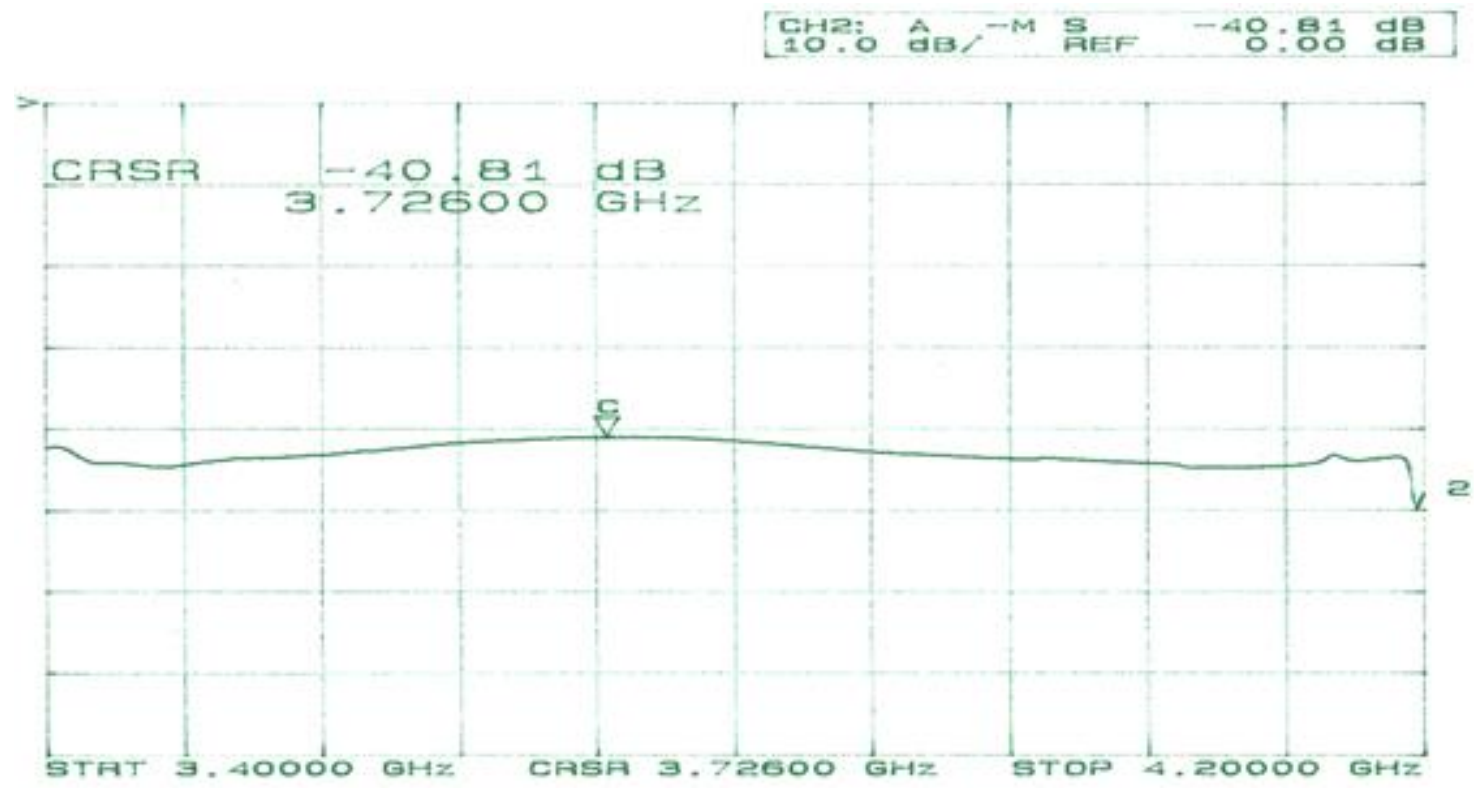

Figure 11: Cross polarisation discrimination at through port (P1)

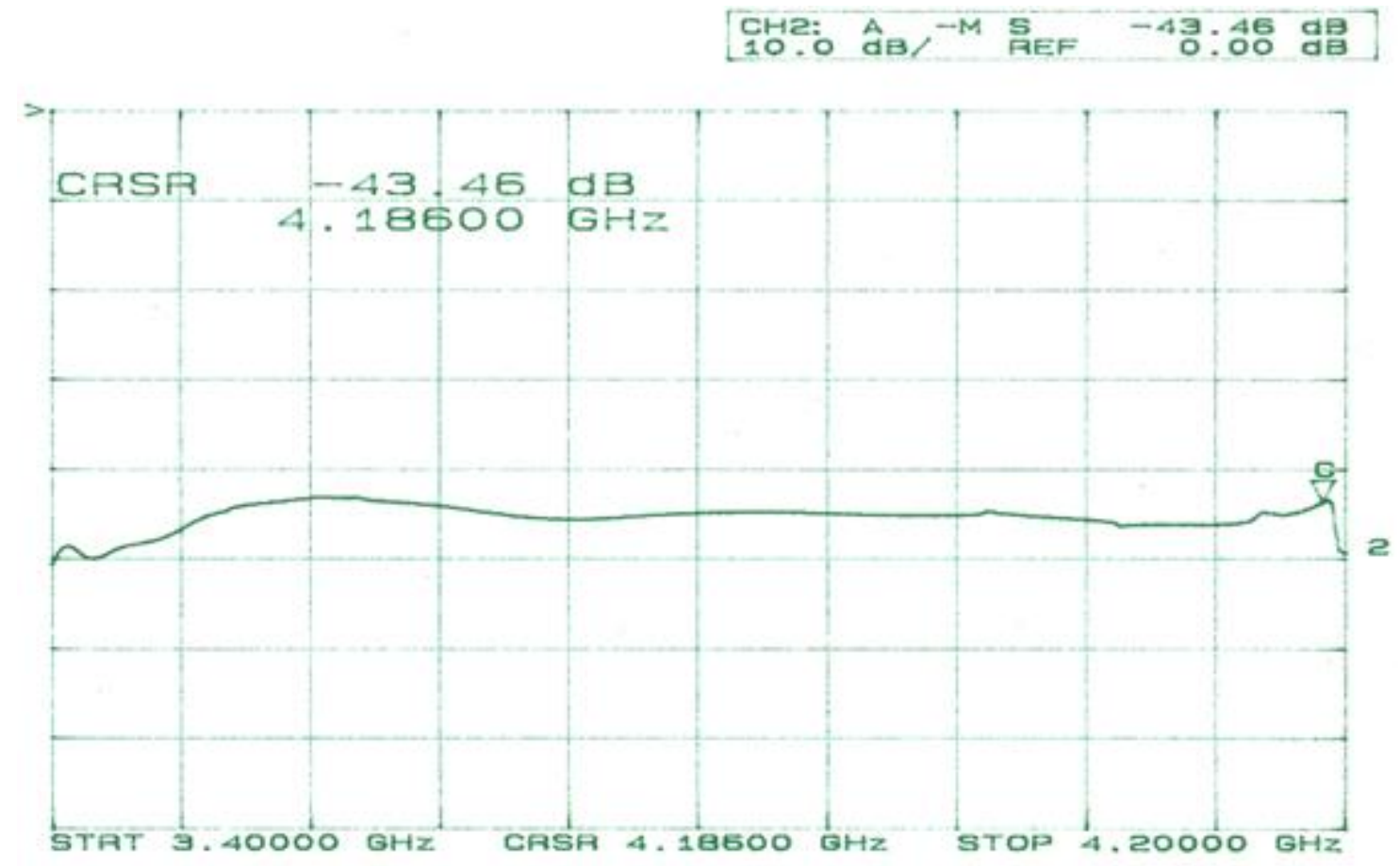

Figure 12: Cross polarization discrimination at side port (P2) 


\section{Conclusion}

A simple and compact C band OMT having a fractional bandwidth of $21 \%$ is being designed, simulated, and manufactured in this paper. Measured results show very good agreement with simulated values. Excellent electrical performance is achieved with return loss of both ports better than $20 \mathrm{~dB}$, port to port isolation greater than $52 \mathrm{~dB}$ and Cross Polarization discrimination better than $40 \mathrm{~dB}$. Mechanical fabrication simplicity to achieve light weight and a compact product is also successfully demonstrated. The insertion loss value measurement was missed out due to unavailability of one more OMT. This design can be adopted for the entire narrow band OMT's of other satellite bands offering simple manufacturing and high performance. It is to be noted that the mechanical butting to be perfect for the split pieces to avoid spikes and performance degradation for the higher band of operation.

\section{ACKNOWLEDGEMENTS}

The author is grateful to A.Venugopal, Head AP\&SD for offering the measurement facility, S.ChinnaBabu for mechanical drawing and fabrication support and Krishnamohan for testing at RF Lab.

\section{REFERENCES}

[1] Stuchly, S., Wide-band rectangular to circular waveguide mode and impedance transformer," IEEE Trans. Microwave Theory and Techniques, Vol. 13, No. 5, 379-380, 1965.

[2] A. M. Boifot, E. Lier, and T. Schaug-Pettersen, Simple and broad band orthomode transducer, IEEE Proc 137 (1990), 396-400.

[3] Boifot, A. M., "Classification of orthomode transducer," European Trans on Emerging Telecommunication Technologies, Vol. 2, No. 5, 503-510, 1991.

[4] N. Yoneda, M. Miyazaki, M. Tanaka, and H. Nakaguro, "Design of compact-size high isolation branching OMT by the mode-matching technique," in Proc. 26th Eur. Microw. Conf., Sep. 1996, pp. $848-851$.

[5] U. Rosenberg and R. Beyer, "Compact T-junction orthomode transducer facilitates easy integration and low cost production," in Proc. 41st Eur. Microw. Conf. (EuMC), Oct. 2011, pp. 663-666.

[6] P. N. Choubey and W. Hong, "Novel wideband orthomode transducer for 70-95 GHz," in Proc. IEEE Int. Wireless Symp. (IWS), Mar./Apr. 2015, pp. 1-4.

[7] C. A. Leal-Sevillano, Y. Tian, M. J. Lancaster, J. A. Ruiz-Cruz, J. R. Montejo-Garai, and J. M. Rebollar, "A micromachined dual-band orthomode transducer," IEEE Trans. Microw. Theory Techn., vol. 62, no. 1, pp. 55-63, Jan. 2014.

[8] H. Schlegel and W. D. Fowler, "The ortho-mode transducer offers a key to polarization diversity in EW systems," Microw. Syst. News, vol. 9, pp. 65-70, Sep. 1984.

[9] BRAIN, J.R.: 'The design and evaluation of a high performance $3 \mathrm{~m}$ antenna for satellite communication', The Marconi Review, 1978, Fourth Quarter, pp. 218-236

[10] A. Navarrini and R. Nesti, "Symmetric reverse-coupling waveguide orthomode transducer for the 3mm band," IEEE Trans. Microw. Theory Techn., vol. 57, no. 1, pp. 80-88, Jan. 2009. 
[11] A. Tribak, J. L. Cano, A. Mediavilla, and M. Boussouis, "Octave bandwidth compact turnstile-based orthomode transducer," IEEE Microw. Wireless Compon. Lett., vol. 20, no. 10, pp. 539-541, Oct. 2010.

A. Navarrini and R. L. Plambeck, “A turnstile junction waveguide orthomode transducer," IEEE Trans. Microw. Theory Techn., vol. 54, no. 1, pp. 272-277, Jan. 2006.

[13] S.-G. Park, H. Lee, and Y.-H. Kim, "A turnstile junction waveguide orthomode transducer for the simultaneous dual polarization radar," in Proc. Asia-Pacific Microw. Conf. (APMC), Dec. 2009, pp. 135-138.

[14] U. Rosenberg, A. Bradt, M. Perelshtein, and P. Bourbonnais, "Broadband ortho-mode transducer for high performance modular feed systems," in Proc. 40th Eur. Microw. Conf. (EuMC), Sep. 2010, pp. 807-810.

[15] Design and Simulation of Orthomode Transducer in Ku-Frequency Band on HFSS, Proceedings ofthe 12th IEEE International Multitopic Conference, December 23-24,2008

[16] Design and Simulation of a Multiband Orthomode Transducer in C Band by Abdellah El Kamili, Angel Mediavilla, Jaouad Terhzaz, Abdelwahed Tribak, Research Gate, 2018

[17] Realization of Ku-Band Ortho Mode Transducer with High Port to Port Isolation by Pramendra K. Verma and Raj Kumar, Progress In Electromagnetics Research Letters, Vol. 74,111-115, 2018

\section{AUTHOR}

Rahul G received his M.E in Microwave and Radar Engineering in the year 2015 from Osmania University, Hyderabad, India. He is currently working in Electronics Corporation of India Limited in the design, development and testing of ground station terminals for Satellite communications. He has designed various feed systems for the Earth station antennas for tracking the GEO and LEO satellites. His areas of interest are waveguide feed system design, reflector designs for satcom airborne antennas, and patch antennas.

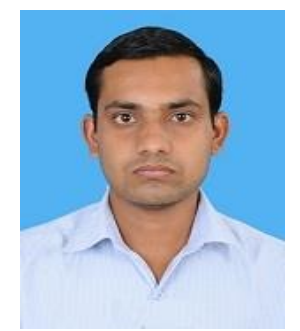

